\title{
Assessment of secondary particles in breast proton therapy by Monte Carlo simulation code using MCNPX
}

\author{
F. Maroufkhani, S.M.M. Abtahi* ${ }^{*}$ T. Kakavand
}

Physics Department, Imam Khomeini International University, Qazvin, Iran

\begin{abstract}
\section{- Original article}

\author{
*Corresponding authors: \\ S.M. Mahdi Abtahi, PhD., \\ E-mail:
}

sm.abtahi@sci.ikiu.ac.ir

Revised: December 2019

Accepted: January 2020

Int. J. Radiat. Res., January 2021; 19(1): $23-29$

DOI: 10.29252/ijrr.19.1.23

Background: The present study aimed to investigate the equivalent dose in vital organs, including heart and lung, due to secondary particles produced during breast proton therapy. Materials and Methods: The numerical ORNL female-phantom was improved and simulated using the Monte Carlo MCNPX code. The depth-dose profile of proton beams with different energies was simulated. The proper energy range of incident proton beams has been estimated in order to have the Bragg peaks inside the breast tissue. The equivalent dose of secondary particles, including neutron and photon in vital organs, were evaluated. The TALYS code was used to investigate the neutron and photon particles' production cross-sections. Results: The results showed that for the proton energy range of $60-70 \mathrm{MeV}$, the Bragg peaks positioned inside the breast. The maximum dose of $0.65 \mathrm{mSv} / \mathrm{nA}-\mathrm{p}$ was in Heart-Left Ventricle due to neutrons production by incident $70 \mathrm{MeV}$ protons. However, the maximum absorbed dose, due to the secondary particles, was less than $0.0004 \%$ of proton equivalent dose at the Bragg peak. The maximum photons dose and the protons dose into the Heart-Left Ventricle were $8.42 \mu \mathrm{Sv} / \mathrm{nA}-\mathrm{p}$ and $68.08 \mu \mathrm{Sv} / \mathrm{nA}-\mathrm{p}$, respectively, which were negligible compared to the proton equivalent dose at the Bragg peak. Conclusion: The results confirmed a noticeable lower dose in the heart and lungs for breast proton therapy, compared with the previously reported dose for breast radiotherapy using photon. Most of the dose absorbed by the organs is due to the secondary neutrons, but those are low enough to be neglected.
\end{abstract}

Keywords: Proton therapy, breast, secondary particles, equivalent dose, MCNPX.

\section{INTRODUCTION}

Cancer is a term in which abnormal cells divide without control and can attack other tissues. According to the World Health Organization, cancer is the worldwide leading death cause for people younger than 85 years (1). According to the estimates of the worldwide incidence and mortality for 27 cancers in 2008, breast cancer is the second most common cancer (10.9\% of all) and the leading cause of cancer death in women worldwide, with more than one million diagnoses in 2008 (2).

On average, breast cancer is responsible for $25 \%$ of cancers that Iranian women suffer from
(3). Treatment techniques for breast cancer vary depending on its kind and stage. The types of treatment are most often based on surgery, radiation therapy, and chemotherapy. These therapies may be used alone or in combination, depending on the stage of the disease. Surgery and radiotherapy are commonly used to treat patients with early-stage breast cancer.

The physical properties of heavy particles, like protons, can, in some cases, allow for an improved dose distribution, compared with conventional X-ray radiotherapy (4). In breast radiotherapy, the heart receives doses of 1 to 5 Gy (5). Previous studies have suggested that exposure to this level can cause ischemic heart 
disease (6, 7). The proton Bragg peak, characterized by a sharp distal penumbra, makes it possible to limit the dose to critical organs in ways that are not possible with X-ray radiotherapy ${ }^{(8)}$. This distinct advantage, coupled with falling costs, has led to a significant increase in the interest in, and availability of proton beam therapy. The interest in proton therapy for breast cancer has substantially increased over the past decades, as evidenced by the recently opened, 1700 patient, randomized trial of proton vs photon therapy for breast cancer patients (9) and the many publications on this subject (10).

In proton therapy, the beam of the proton is projected accurately towards the cancerous cells. Hence, for breast cancer patients, proton therapy has the potential to spare more normal tissue than other conventional radiation therapy. So, it leads to a fewer risk of side effects, especially to the heart and lung. However, due to the high energy protons used in proton therapy, the effect of the produced secondary particle should be investigated. When protons travel through matter, secondary particles like neutrons and photons, are produced by the interactions of protons within the patient's body. The production of neutrons and photons by the primary proton beam, however, could be a significant contribution to the integral dose, and thus diminish this potential advantage. Also, neutrons have a large quality factor, and thus, even a small physical dose can result in essential biological effects (11).

It is believed that the secondary dose has no known beneficial effect and can lead to secondary cancer ${ }^{(12)}$. For women with left-sided breast cancer, it's very important to deliver the lowest dose to vital organs, like heart and lung. The side effects of proton therapy for breast cancer has not been supported by adequate patient data. Furthermore, data on the relative biological effect on different tumor cells and normal tissue cells under proton irradiation remain scarce.

The current study aimed to investigate the equivalent dose in vital organs, including heart and lungs, due to secondary particles produced during the breast proton therapy. To this end, an optimum proton energy range was determined and the equivalent dose of secondary particles was investigated as a function of incident proton energy.

\section{MATERIALS AND METHODS}

\section{The ORNL phantom material improvement}

The Monte Carlo simulations were done using MCNPX (Monte Carlo N-particles eXtension) version 2.6, which can handle the interaction and transport of protons, neutrons, electrons, and other particles over a vast range of energies (13). In the current study, to investigate the analytical phantom of the human body, based on ORNL publications (14), ORNL-female phantom was used to create the input file for the MCNPX transport code. Figure 1 illustrates the scheme of this phantom.

In the original ORNL phantom, the breast tissue was considered as soft tissue, and for all soft tissues, a unique material had been used. However, it is well known that the material composition of breast tissue is different from other soft tissues. Hence, the breast material was improved. The breast material composition used in the current study for ORNL phantom is presented in table $1(15,16)$. The mass densities of all tissues were also improved based on the data presented in table $2{ }^{(15) .}$

Table 1. The elemental composition of the breast tissue ${ }^{(15,}$

16), the normalized weight fraction is demonstrated.

\begin{tabular}{|c|c|c|c|c|c|c|c|c|c|}
\hline organ & $\begin{array}{c}\text { Density } \\
\left(\mathbf{g} / \mathbf{c m}^{\mathbf{3}}\right)\end{array}$ & $\mathbf{H}$ & $\mathbf{C}$ & $\mathbf{N}$ & $\mathbf{O}$ & $\mathbf{N a}$ & $\mathbf{S}$ & $\mathbf{P}$ & $\mathbf{C l}$ \\
\hline Breast & 1.02 & 0.106 & 0.332 & 0.030 & 0.527 & 0.001 & 0.002 & 0.001 & 0.001 \\
\hline
\end{tabular}

Table 2. The mass density of the tissues used to improve the ORNL- female phantom ${ }^{(15)}$.

\begin{tabular}{|c|c|}
\hline Density $\left(\mathrm{g} / \mathrm{cm}^{\mathbf{3}}\right)$ & Materials \\
\hline 0.00129 & Air \\
\hline 1.04 & Soft tissue \\
\hline 0.296 & Lung \\
\hline 1.4 & Bone \\
\hline 1.02 & Breast \\
\hline
\end{tabular}

Int. J. Radiat. Res., Vol. 19 No. 1, January 2021 


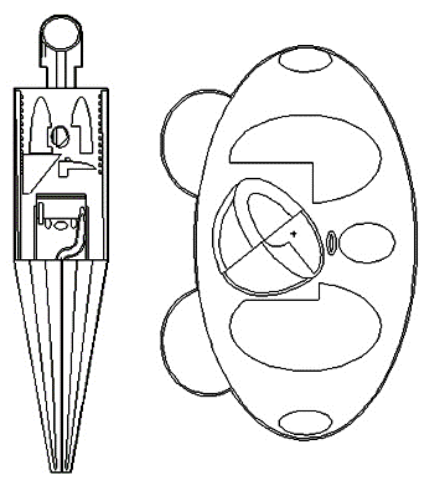

Figure 1. Left: Longitude crosses of ORNL phantom. Right: Transversal cross-sections of an adult female phantom ${ }^{(14)}$. Heart and lungs are visible in the figure.

\section{The applicable proton energy range}

In the current study, the energy range of the proton beam used for breast proton therapy was calculated. To this aim, the depth-dose profiles of proton beams with different energies were simulated. At each energy, a proton beam with a circular field of $4 \mathrm{~cm}$ diameter was simulated. Six energies of $60,62,64,68$, and $70 \mathrm{MeV}$ for incident protons were investigated. Afterward, at each energy, the depth-dose curves were investigated and the positions of the related Bragg peaks were evaluated.

The range of incident proton was determined so that the maximum proton dose was not absorbed beyond the breast. Twenty-four spherical cells of $1 \mathrm{~mm}$ radius were simulated on the beam penetration axis, centered at the depths of $2.5 \mathrm{~mm}$ to $48.5 \mathrm{~mm}$, to simulate the dose of the proton as a function of depth. The adjacent tally cells were tangent to each other. Hence, the distance between the centers of two adjacent spherical tally cells was $2 \mathrm{~mm}$. The dose of proton in each cell was calculated using F6 tally. It should be noted that the F6 tally calculated the energy deposition, which can be converted to the absorbed dose. The quality factor for high energy protons was reported 10 (17). Hence, to convert the absorbed dose of the proton to the equivalent dose, the results were multiplied by a factor of 10 . The MCNPX tallies are normalized per source particle. Furthermore, the unit of F6 tally card in MCNPX code is $\mathrm{MeV} / \mathrm{g}$. In this study, the equivalent dose (in Sv unit) per nano-ampere (1nA) of incident protons was calculated. The normalization factor was calculated according to the equation 1 .

$$
\begin{aligned}
& N F=\frac{1 / \mathrm{Kg}}{\mathrm{MeV} / g} \times \frac{\mathrm{nA} / \mathrm{A}}{\text { Electericcharge of a proton }} \times Q F= \\
& 1.6 \times 10^{-10} \times 0.625 \times 10^{10} \times 1010
\end{aligned}
$$

The first fraction in eq.1 converts the rest of the F6 tally to the SI unit of Gy. The second fraction calculates the required number of source particles for $1 \mathrm{nA}$ of the proton. $\mathrm{QF}$ is the quality factor.

\section{Track of secondary particles}

As protons moving inside the matter undergo inelastic nuclear interactions, secondary particles, such as neutrons, photons, secondary protons, deuterons, are produced (18). In the energy range used for proton therapy, neutrons and photons are the most important secondary particles, because they can travel far distances from the target tissue and store their energies in other organs, thereby increasing the risk of side effects $(19,20)$. In the MCNPX, the energy of the residual nuclei and the non-traced particles are considered to be deposited locally at the point of production.

To achieve more accurate results, all predicted produced particles were tracked. Hence, in the current study, protons, neutrons, and photons were transported throughout the simulated geometry. In the worst-case scenario, the irradiation should be performed for the left breast. Hence, the cyclotron proton source, as a directional point source in the vacuum space, was modeled in front of the left breast of the ORNL-female phantom.

The secondary particle doses were simulated as a function of proton energy in an energy range of $60-70 \mathrm{MeV}$. The rationale behind the selection of this energy range was based on the result of the energy range applicable for breast proton therapy. The F4 tally card was used to calculate the dose of transported neutron and photon particles and the dose function (DF) card for equivalent dose. The flux to dose conversion factors was according to the report of ANSI/ANS -6.1.1-1977 (21).

The equivalent dose of secondary particles per $1 \mathrm{nA}$ of the incident proton was calculated. 
The controls unit parameters of the DF card were sets to the SI unit of Sievert (Sv) for equivalent dose. Since, for protons, the flux to dose conversion factors was not reported in available standards, the F6 card was used to energy deposition calculation. The energy deposition was converted to the equivalent dose.

To investigate the neutron and photon production probability, the total neutron, as well as photon production cross-sections, were calculated for the proton interaction with oxygen, carbon, and nitrogen. It should be noted that other elemental compositions have low weight in secondary particles production in breast tissue. Hence, they were ignored from cross-section calculation. To calculate the $(\mathrm{p}, \mathrm{n})$ and $(\mathrm{p}, \gamma)$ cross-sections, the talys code (22) was used. To investigate the effect of energy on the cross-section, a wide range of proton energy (60 $-200 \mathrm{MeV}$ ) was investigated.

\section{RESULTS}

\section{Bragg peak investigation in breast tissue}

The curves of proton depth-dose profiles in breast tissue for different proton energies are demonstrated in figure 2 .

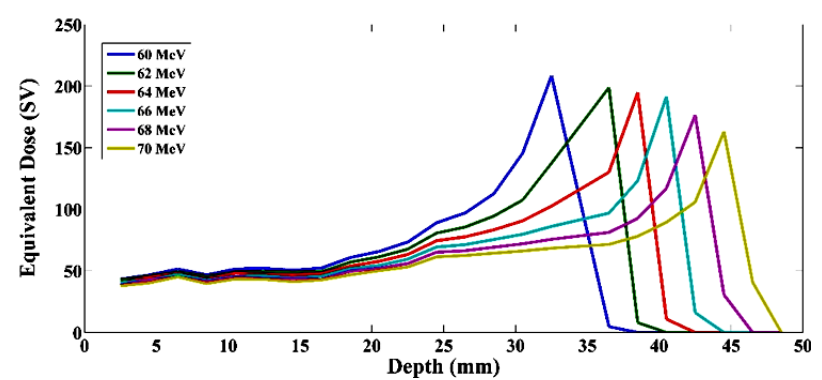

Figure 2. Depth dose profiles in the breast of ORNL-female phantom for 1nA incident proton with an energy range of 60$70 \mathrm{MeV}$ on the left breast. A specific color demonstrates the results of each proton energy.

It can be seen that, for this arrangement, the Bragg peaks fall inside the breast. All tally errors were less than $5 \%$. The results showed that as the proton energy increases, the particle penetration power increases. This result is in agreement with the Bragg-Kleeman rule equation 2 (23).

$\mathrm{R}=\alpha \mathrm{Ep}$

Where $\alpha$ is the proportionality factor, which is determined by $0.0022 \mathrm{~cm} \cdot \mathrm{MeV}-1 . \mathrm{P}$ is the exponent of range-energy relation that its value was reported 1.77 for proton transport in water. However, as the number of non-elastic nuclear reactions with the atomic nucleus rises, the Bragg peak height decreases.

\section{Equivalent dose of secondary particles}

Figure 3 shows the equivalent doses of secondary photons, neutrons and protons in vital tissues of the improved ORNL-female phantom per $1 \mathrm{nA}$ of incident proton beams.
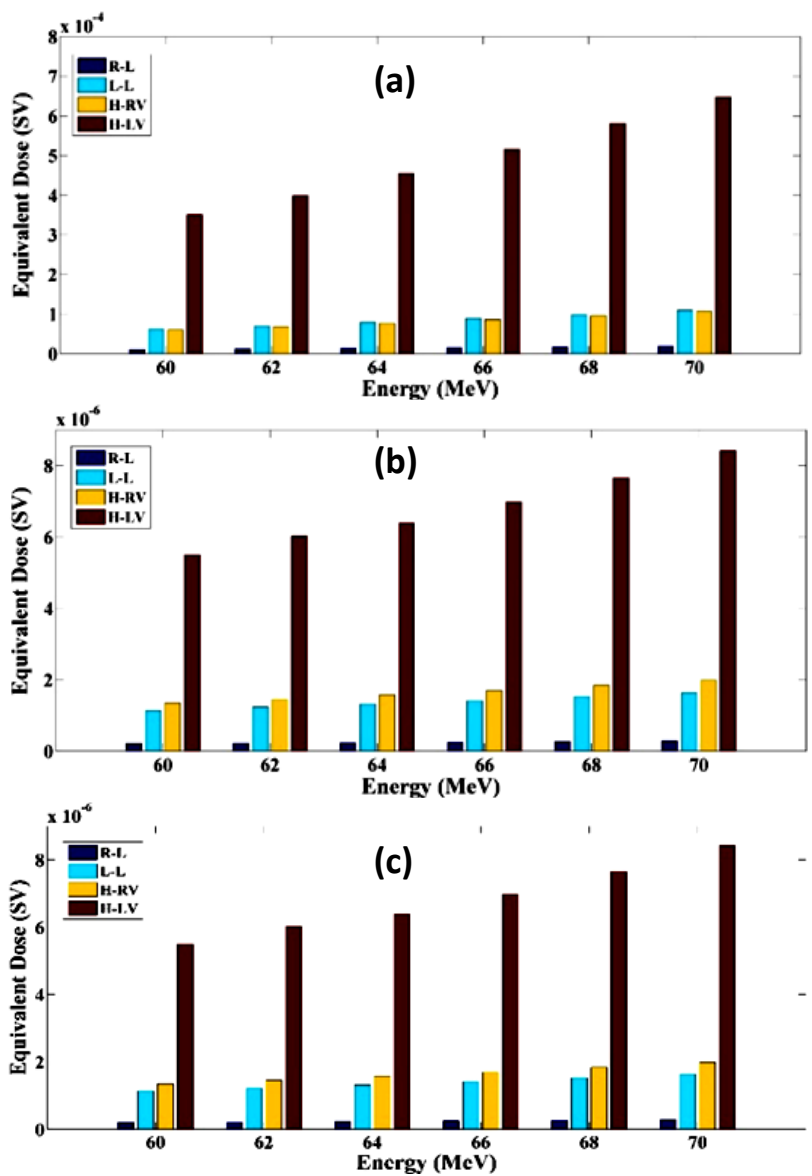

Figure 3. Secondary Equivalent Dose (SV) per 1nA of the incident proton in vital organs for six different proton beam energies. (a) Neutron dose equivalent; (b) Photon dose equivalent; (c) Proton dose equivalent. (Right-Lung (R-L), Left-Lung (L-L), Heart-Right Ventricle (H-RV), Heart-Left Ventricle (H-LV)).

Int. J. Radiat. Res., Vol. 19 No. 1, January 2021 
The results demonstrated in figure 3 showed that the equivalent dose of secondary particles in the organs increases with the beam energy. The Heart Left Ventricle (HLV) had the highest equivalent dose $(H)$. The neutron equivalent dose in $\mathrm{HLV}$ increases from $0.35 \mathrm{mSV} / \mathrm{nA}$ for the incident proton energy of $60 \mathrm{MeV}$ to $0.65 \mathrm{mSV} /$ $\mathrm{nA}$ for the incident proton energy of $70 \mathrm{MeV}$. The photon and proton equivalent doses range from $5.50 \mu \mathrm{SV} / \mathrm{nA}$ to $8.42 \mu \mathrm{SV} / \mathrm{nA}$ and $38.00 \mu \mathrm{SV} / \mathrm{nA}$ to $68.08 \mu \mathrm{SV} / \mathrm{nA}$ at an energy range of $60-70 \mathrm{Mev}$, respectively. In the current study, the composition of the breast tissue was improved.

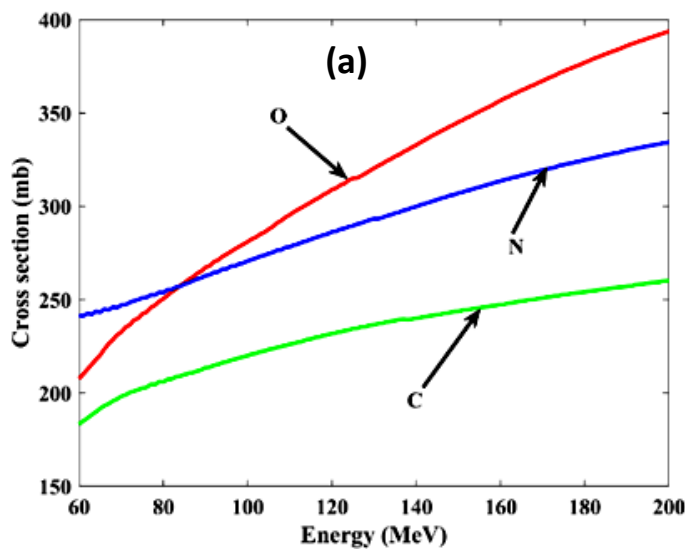

Figure 4. (a) Total neutron production cross-section as a function of energy for the incident proton on carbon, oxygen and nitrogen; (b) Total photon production cross-section as a function of energy for the incident proton on carbon, oxygen, and nitrogen.

\section{DISCUSSION}

The results showed that the Bragg peak depth increased with the increase of incident proton energy. However, the height of the Bragg peak decreases as the proton energy increases. This finding was following the theoretical model reported by Ulmer and Matsinos (24). They theoretically analyzed an impinging proton beam lets on the water using the GEANT4 Monte Carlo simulation code. They found that the absorbed energy at the Bragg peak of the proton was more than $75 \mathrm{MeV} \cdot \mathrm{cm}^{2} / \mathrm{g}$ for the proton energy of $50 \mathrm{MeV}$, which decreased to less than $50 \mathrm{MeV} . \mathrm{cm}^{2} / \mathrm{g}$ for the proton energy of $100 \mathrm{MeV}$. In another theoretical study, Thomas Bortfeld proved that the Bragg peak height decreases as the proton energy increases (23).

In a study, Jia et al. reported the depth dose

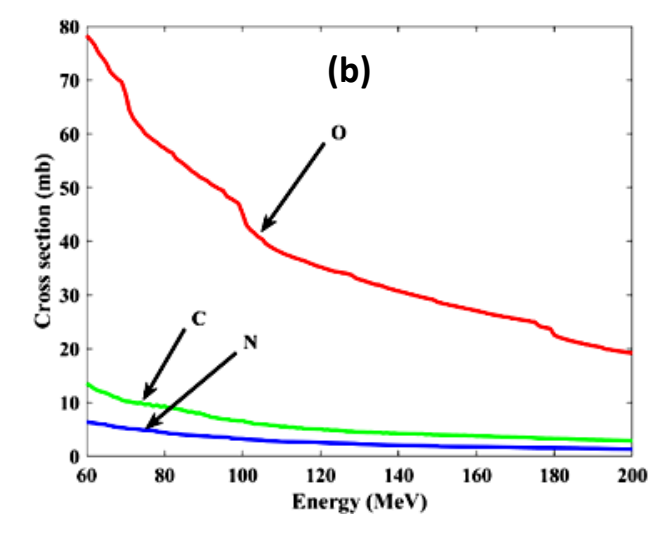

The cross-sectional investigation showed the importance of rectified breast material composition in the investigation of the secondary particle. The cross-sections of the neutrons and photons production for the proton interaction with three main components of the breast tissue are shown in figures 4 (a) and (b), respectively. Furthermore, the results of figure 4 showed the effect of incident proton energy in the secondary particle production. The results showed that the neutron and photon production probabilities were increased with the increase of incident proton energy. profiles in the slab head phantom for the incident pencil beams using the Monte Carlo MCNPX code (4). They reported an absorbed dose of about $0.048 \mathrm{nGy} /$ particle at the Bragg peak of the proton beam with the energy of $60 \mathrm{MeV}$. They found that the absorbed dose decreased to about $0.042 \mathrm{nGy} /$ particle at the Bragg peak when the proton energy increased to $70 \mathrm{MeV}$. The finding of Jia et al. is comparable with the result of the current study. In the present research, a decrease of $21 \%$ in the Bragg peak height was accrued when the proton energy increased from $60 \mathrm{MeV}$ to $70 \mathrm{MeV}$. The difference between these two findings was attributed to the difference between the nuclear interaction of protons with the material composition of the brain and breast.

Regarding the secondary particles, the results showed that the maximum neutron equivalent 
dose is in Heart-Left Ventricle. However, the maximum equivalent dose was less than $1 \mathrm{mSv} /$ $\mathrm{nA}$ for the incident proton energy up to $70 \mathrm{MeV}$. The outcomes exhibited that the equivalent doses of gamma were noticeably less than those of neutron in the considered organs. A cross-section study explains this result. It was found that photons in comparison with neutrons, have a lower amount of production cross-section, in the interaction of proton with the components of breast tissue.

Furthermore, the quality factors of the neutron are more than that of the photon. Besides, the attenuation of produced photons was more pronounced than that of neutrons in tissues. The results of the current study are comparable with that reported by Agosteo et al. (25). They investigated the neutron and photon doses in eye proton therapy due to three existing proton therapy facilities at the Bragg peak position using the Monte Carlo Fluka code. They found the doses of secondary particles produced in a passive beam ranged between $10^{-4}$ to $10^{-2}$ Gy per therapy Gy.

In current study, the effect of secondary particles on the non-target organs was investigated. Hence, as expected, the number of secondary particles was less than that obtained by Agosteo and his colleagues. In another study, to investigate the effects of the secondary particles in the proton therapy, the dose of secondary particles produced during the irradiation of a water phantom by proton beam energies of $200 \mathrm{MeV}$ and $250 \mathrm{MeV}$ were evaluated using Monte Carlo method (26).

Also, Schneider et al. measured the secondary neutron dose during the irradiation of a water phantom with protons using Monte Carlo simulations by the FLUKA code (27). They showed that the neutron dose equivalent could be reached for a medium-sized target volume of approximately $1 \%$ of the treatment dose, and the dose deposited by the secondary neutrons during proton radiotherapy using the spot scanning technique can be neglected in the treatment region. However, as shown by the results of the current study, the cross-sections of secondary particles depend on the material composition. Hence, the production of the secondary particle is a function of the elemental composition of the target.

Furthermore, the neutron penetration in different compounds strongly depends on their compositions (28). The results demonstrated that the equivalent dose, due to the secondary particles in different organs, raised with the increasing of incident proton energy. This consequence is in accordance with that reported by Farah et al. (29). They reported that increasing the proton-induced the neutron dose of non-target organs by increasing the proton energy in the proton therapy for the craniopharyngioma and ocular melanoma. Also the maximum equivalent dose was related to the Heart-Left Ventricle, due to the irradiation to the left breast, whereas the minimum equivalent dose was related to Right-Lung. The equivalent doses for Left-Lung and Heart-Right Ventricle are almost the same.

The meta-analysis of dosimetric studies revealed both statistically and a clinically significant decrease in the lung and heart doses with proton beam plans in comparison with photon plans (30). The results of the current study confirmed this statement.

\section{CONCLUSION}

The equivalent doses of secondary neutrons and photons in vital organs were calculated for breast proton therapy. The results confirmed a noticeable lower dose in heart and lung for the breast proton therapy compared with the previously reported dose for breast radiotherapy using photon. Heart-Left Ventricle (HLV) received the maximum dose from secondary particles. However, the dose was less than $1 \mathrm{mSv}$. Most of the dose absorbed by the organs is due to the secondary neutrons, but those are low enough to be neglected.

Conflicts of interest: Declared none. 


\section{REFERENCES}

1. Thariat J, Hannoun-Levi JM, Myint AS, Vuong T, Gérard JP (2013) Past, present, and future of radiotherapy for the benefit of patients. Nature Reviews Clinical Oncology, 10 (1): 52.

2. Ferlay J, Shin HR, Bray F, Forman D, Mathers C, Parkin DM (2010) Estimates of worldwide burden of cancer in 2008: GLOBOCAN 2008. Int J cancer, 127(12): 2893-917.

3. Harirchi I, Karbakhsh M, Kashefi A, Momtahen AJ (2004) Breast cancer in Iran: results of a multi-center study. Asian Pacific Journal of Cancer Prevention, 5(1): 24-7.

4. Jia SB, Mowlavi AA, Hadizadeh MH, Ebrahimi Loushab M (2014) Impact of range straggling and multiple scattering on proton therapy of brain, using a slab head phantom. Int $J$ Radiat Res, 12(2): 161-7.

5. Piroth MD, Baumann R, Budach W, Dunst J, Feyer P, Fietkau $R$, et al. (2019) Heart toxicity from breast cancer radiotherapy : Current findings, assessment, and prevention. Strahlentherapie und Onkologie, 195(1): 1-12.

6. Shimizu Y, Kodama K, Nishi N, Kasagi F, Suyama A, Soda M, et al. (2010) Radiation exposure and circulatory disease risk: Hiroshima and Nagasaki atomic bomb survivor data, 1950-2003. BMJ, 340: b5349.

7. Azizova T, Muirhead C, Druzhinina M, Grigoryeva E, Vlasenko E, Sumina M, et al. (2010) Cardiovascular diseases in the cohort of workers first employed at Mayak PA in 1948-1958. Radiat Res, 174(2): 155-68.

8. Esmaili Torshabi A (2013) Investigation of tumor motion influence on applied dose distribution in conventional proton therapy vs. IMPT a 4D Monte Carlo simulation study. Int J Radiat Res, 11(4): 225-31.

9. Bekelman JE, Lu H, Pugh S, Baker K, Berg CD, de Gonzalez $A B$, et al. (2019) Pragmatic randomised clinical trial of proton versus photon therapy for patients with nonmetastatic breast cancer: the Radiotherapy Comparative Effectiveness (RadComp) Consortium trial protocol. BMJ open, 9(10): e025556.

10. Kammerer E, Le Guevelou J, Chaikh A, Danhier S, Geffrelot J, Levy C, et al. (2018) Proton therapy for locally advanced breast cancer: a systematic review of the literature. Cancer Treatment Reviews, 63:19-27.

11. Cember H, Johnson TE. (2010) Introduction to health Physics edition F, editor. New York: McGraw-Hill Companies, Inc. 2010.

12. Newhauser $\mathrm{W}$, Fontenot J, Zheng $\mathrm{Y}$, Polf J, Titt $\mathrm{U}$, Koch $\mathrm{N}$, et al. (2007) Monte Carlo simulations for configuring and testing an analytical proton dose-calculation algorithm. Physics in Medicine \& Biology, 52(15): 4569.

13. Pelowitz DB (2005) MCNPXTM user's manual. Los Alamos National Laboratory, Los Alamos. 2005.

14. Eckerman K, Cristy M, Ryman J (1996) The ORNL mathematical phantom series. Oak Ridge, TN: Oak Ridge National Laboratory. 1996.

15. Jr RM, Gesh C, Pagh R, Rucker R, Williams R (2011) Compendium of material composition data for radiation transport modeling. Richland, Washington: U.S. depart- ment of homeland security U.S. customs and border protection and domestic nuclear detection office under U.S. Department of Energy, 2011 Contract No.: PNNL-15870 Rev. 1.

16. Goldstone K (1990) Tissue Substitutes in Radiation Dosimetry and Measurement, in: ICRU Report 44, International Commission on Radiation Units and Measurements, USA (1989). WB Saunders; 1990.

17. Commission USNR (2014) NRC: 10 CFR 20.1004 Units of radiation dose.

18. Kraft G (2000) Tumor therapy with heavy charged particles. Progress in Particle and Nuclear Physics, 45: S473S544.

19. Taddei PJ, Mirkovic D, Fontenot JD, Giebeler A, Zheng $Y$, Kornguth D, et al. (2009) Stray radiation dose and second cancer risk for a pediatric patient receiving craniospinal irradiation with proton beams. Physics in Medicine \& Biology, 54(8): 2259.

20. Jiang $H$, Wang $B, X u X G$, Suit HD, Paganetti H (2005) Simulation of organ-specific patient effective dose due to secondary neutrons in proton radiation treatment. Physics in Medicine \& Biology, 50(18): 4337.

21. Society AN (1997) American National Standard Neutron and Gamma-Ray Flux-to-Dose Rate Factors (N666). LaGrange Park: ANSI/ANS- 6.1.1.

22. Koning A, Hilaire S, Goriely S (2015) TALYS-1.8 : A nuclear reaction program, user manual. Netherlands: 2015 Contract No.: NRG-1755 ZG PETTEN.

23. Bortfeld T (1997) An analytical approximation of the Bragg curve for therapeutic proton beams. Med Phys, 24(12): 2024-33.

24. Ulmer W and Matsinos E (2010) Theoretical methods for the calculation of Bragg curves and 3D distributions of proton beams. The European Physical Journal Special Topics, 190(1): 1-81.

25. Agosteo S, Birattari C, Caravaggio M, Silari M, Tosi G (1998) Secondary neutron and photon dose in proton therapy. Radiotherapy and oncology, 48(3): 293-305.

26. Didi A, Dekhissi H, Sebihi R, Krim M, Mohamed RM (2019) Calculate Primary and Secondary Dose in Proton Therapy Using 200 and $250 \mathrm{MeV}$ Proton Beam Energy. Moscow University Physics Bulletin, 74(4): 364-8.

27. Schneider U, Agosteo S, Pedroni E, Besserer J (2002) Secondary neutron dose during proton therapy using spot scanning. Int J Radiat Oncol Biolphys, 53(1): 244-51.

28. Abtahi SM, Aghamiri SMR, Khalafi H, Rahmani F (2014) An investigation into the potential applicability of gel dosimeters for dosimetry in boron neutron capture therapy. Int $J$ Radiat Res, 12(2):149-59.

29. Farah J, Sayah R, Martinetti F, Donadille L, Lacoste V, Herault J, et al.(2014) Secondary neutron doses in proton therapy treatments of ocular melanoma and craniopharyngioma. Radiat Protect Dosi, 161(1-4): 363-7.

30. Tian G, Li N, Li G (2013) Dosimetric comparing between protons beam and photons beam for lung cancer radiotherapy: a meta-analysis. Editorial office of Chinese Journal of Lung Cancer, 16(5): 252-60. 
